\title{
Interference Modeling and Analysis for Inclined Projective Multiple Beams of GEO Satellite Communication Systems
}

\author{
Wei Zheng, Bin Li, Shubo Ren, Jiang Chen, Jianjun Wu \\ Institution of Advanced Communications, EECS, Peking University, Beijing, 100871, China \\ E-mail: selina@pku.edu.cn
}

\begin{abstract}
In multi-beam satellite systems, Inter-Beam Interference (IBI) has a great effect on system performance. Within range of satellite coverage area, inclined projective multiple beams give rise to elliptic beam projections, resulting in that the distribution of beam projections is different from traditional cellular distribution. In this paper, an Inclined Projection (IP) model is proposed for IBI in OFDMA based GEO satellite communication systems, and corresponding analysis is also included. A comparison of carrier to interference power ratio (C/I) of users at the center of each cell with and without the consideration of IP is made, based on the simulation using different Frequency Reuse (FR) factor. The corresponding conclusions are presented. This research can provide reference for later satellite beam planning.
\end{abstract}

Keywords-inclined projection; interference; multiple beam; satellite

\section{INTRODUCTION}

For the past few years, satellite communications have drawn more and more attention. Orthogonal Frequency Division Multiple Access (OFDMA) scheme is a prospective candidate for satellite communications, and relevant research is in progress [1-3]. In wireless communication systems, system capacity is mainly restricted to limited spectrum [4]. In terrestrial systems, different cells can employ the same frequency to increase spectrum efficiency. In satellite systems, the Frequency Reuse (FR) is accomplished by using multiple beams [5]. Multi-beam satellite systems use different spot beams to distinguish different regions which are in the same satellite coverage area.

In multi-beam satellite communication systems, on-board antenna acts as a spatial filter, leading to a different interference scenario compared to that of terrestrial systems. Interference analysis for multi-beam satellite systems has been widely researched recently, and different interference models for various application scenarios have been established [6-8]. Most are based on assuming beams as circles of the same size. However, due to great satellite coverage area, except the Orthographic Projection (OP) of the satellite, all other cells are approximate ellipses in actual systems.

When a cell is far from the sub-satellite point, its area is much larger than that of the circular cell which is centered on the sub-satellite point. Therefore, if all beam projections in the satellite coverage area are assumed as circular cells which are OPs of the satellite, there will be a large overlap area between actual projections of beams, resulting in bigger actual IBI than assumed. In this paper, an IP model is proposed for IBI in OFDMA based GEO satellite communication systems, and taking a multi-beam satellite system covering Chinese Mainland (CM) for example, C/I of users at different latitude and longitude is calculated.

This paper is organized as follows: Section II describes the system model of multi-beam satellite systems. Section III proposes an IP model for IBI. In Section IV, simplification of $\mathrm{C} / \mathrm{I}$ formula for satellite link is introduced. $\mathrm{C} / \mathrm{I}$ of users in several typical positions is calculated and analyzed in Section V. Section VI concludes the paper.

\section{System Model of Multi-Beam SATEllite SYSTEMS}

In multi-beam satellite communication systems, the satellite coverage area is divided into many regions, each of which is served by a satellite spot beam. On the earth surface covered by a spot beam, the boundary is a level contour. Generally, when planning network, the level of beam boundary is set by a $3 \mathrm{~dB}$ decrease of antenna gain [9].

Research about IBI analysis usually regards on the earth surface as a plane surface. A cell is approximately considered as the OP of the satellite beam. Thus, the cell and the beam form a cone, of which the radius of bottom is the distance corresponding to the half $3 \mathrm{~dB}$ beamwidth. However, in actual systems, most cells are IPs of the satellite, causing the bottom of the cone is an ellipse. The shape of a cell varies with the angle between the beam boresight and the ground. If the beam boresight is perpendicular to the ground, the cell is a circle, as shown in Fig. 1(a), where $\theta_{3 \mathrm{~dB}}$ is half $3 \mathrm{~dB}$ beamwidth. Otherwise, the cell is an ellipse, as shown in Fig. 1(b).

In addition, the smaller the acute angle between the beam boresight and the ground is, the larger the ellipse eccentricity

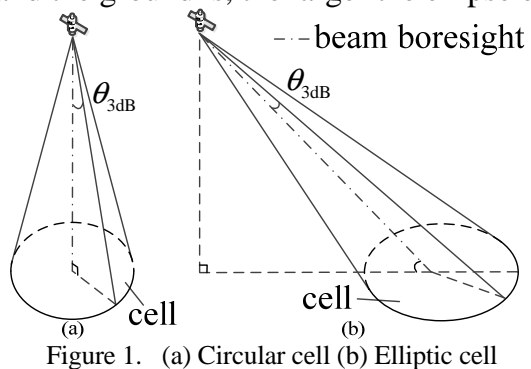


is and hence the ellipse is flatter. It's worth noting that the center of the elliptic cell (the point corresponding to beam boresight) is not the geometric center of the ellipse.

As shown in Fig. 2, this paper is based on a GEO satellite communication system covering $\mathrm{CM}$ by 88 spot beams. The radius of each beam is about $240 \mathrm{~km}$, and the half $3 \mathrm{~dB}$ beamwidth is about $0.38^{\circ}$. After taking IP into consideration, actual shape of each beam projection is shown in Fig. 2, when the GEO satellite is at $110^{\circ} \mathrm{E}$. It can be seen that when a cell is near the sub-satellite point (shown as a red cross in Fig. 2), the shape of elliptic beam projection is like that of the circular beam projection and the overlap between beams is small. On the contrary, when a cell is far from the subsatellite point, the shape of elliptic beam projection is much larger than that of the circular beam projection, and the overlap between beams is large. Therefore, if using the circular beam planning scheme shown in Fig. 2, there will be a large overlap area between actual beam projections, leading to bigger actual IBI than assumed.

\section{INTERFERENCE MODELING OF INCLINED PROJECTIVE MULTIPLE BEAMS}

In terrestrial communication systems, interference is strongly distance-dependent, and its evaluation can usually be limited to the first tier of the neighbor cells or less, if particular and favourable orography is present [6]. In mobile satellite communication systems, on-board antenna acts as a spatial filter and the angular selectivity of beams is not very ideal, so the level of interference depends on the user angular separation, referred to the satellite position (see Fig. 3).

In order to model the interference scenario, the angular separation needs to be calculated first. The calculation method is different with and without the consideration of IP. Taking user $u$ in cell $c$ (represented by $(u, c)$ ) for example, the calculation method of the angular separation $\theta_{u c}^{n}$ (the angle between the boresight of cell $n$ and the line between user $(u, c)$ and the satellite) is explained below.

As shown in Fig. 3, the orbital height of the satellite is registered as $h$, and the earth radius is registered as $R$. $\left(\mathrm{lat}_{S}, \mathrm{lon}_{S}\right),\left(\mathrm{lat}_{p}, \mathrm{lon}_{p}\right)$, $\left(\mathrm{lat}_{u c}, \mathrm{lon}_{u c}\right)$, and $\left(\mathrm{lat}_{n}, \mathrm{lon}_{n}\right)$ represent the latitude and longitude of the GEO satellite orbit, the subsatellite point, user $(u, c)$, and the center of cell $n$, respectively.

When not considering IP (see Fig. 3(a)), the sub-satellite point and the center of cell $n$ are in the same position, $\left(\operatorname{lat}_{p}, \operatorname{lon}_{p}\right)=\left(\operatorname{lat}_{n}, \operatorname{lon}_{n}\right)$, and the satellite, the earth's core, user $(u, c)$ and the center of cell $n$ form a triangle. $\theta_{u c}^{n}$ is given by
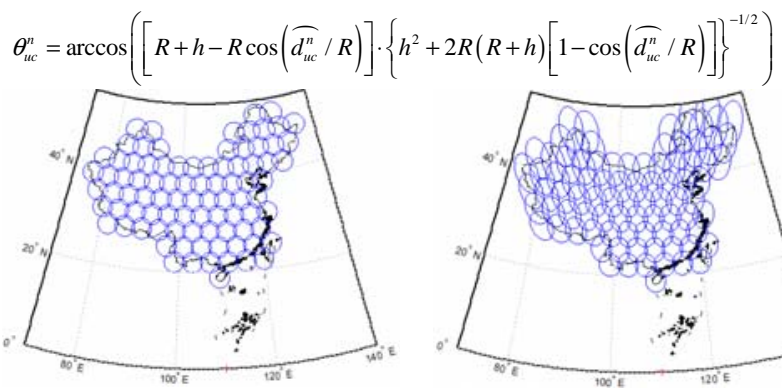

Figure 2. 88 circular and inclined projective spot beams covering CM where $\overparen{d_{u c}^{n}}$ is the spherical distance between user $(u, c)$ and the center of cell $n$,

$$
\widehat{d_{u c}^{n}}=2 R \cdot \arcsin \left(\left\{\begin{array}{l}
\sin ^{2}\left[\left(\operatorname{lat}_{u c}-\operatorname{lat}_{n}\right) / 2\right]+ \\
\cos \left(\operatorname{lon}_{u c}\right) \cos \left(\operatorname{lon}_{n}\right) \sin ^{2}\left[\left(\operatorname{lon}_{u c}-\operatorname{lon}_{n}\right) / 2\right]
\end{array}\right\}\right)
$$

When taking IP into consideration (see Fig. 3(b)), the sub-satellite point and the center of cell $\mathrm{n}$ do not overlap, $\left(\operatorname{lat}_{p}, \operatorname{lon}_{p}\right)=\left(\operatorname{lat}_{S}, \operatorname{lon}_{S}\right)$, and the satellite, the earth's core, user $(u, c)$, and the center of cell $n$ form a tetrahedron. The complexity of calculating $\theta_{u c}^{n}$ increases. $d_{n}^{s}$ (the distance between the cell of center $\mathrm{n}$ and the satellite) and $d_{u c}^{s}$ (the distance between user $(u, c)$ and the satellite) need to be calculated first in order to calculate $\theta_{u c}^{n}$.

$$
\begin{aligned}
& d_{n}^{S}=\left\{h^{2}+2 R(R+h)\left[1-\cos \left(\overparen{d_{n}^{p}} / R\right)\right]\right\}^{1 / 2} \\
& d_{u c}^{S}=\left\{h^{2}+2 R(R+h)\left[1-\cos \left(\widehat{d_{u c}^{p}} / R\right)\right]\right\}^{1 / 2}
\end{aligned}
$$

where $\overparen{d_{n}^{p}}$ is the spherical distance between the center of cell $n$ and the sub-satellite point and $\overparen{d_{u c}^{p}}$ is the spherical distance between user $(u, c)$ and the sub-satellite point.

Then, using the triangle formed by the satellite, user $(u, c)$ and cell $n, \theta_{u c}^{n}$ is given by

$$
\theta_{u c}^{n}=\arccos \left(\left\{\left(d_{n}^{S}\right)^{2}+\left(d_{u c}^{S}\right)^{2}-2 R^{2}\left[1-\cos \left(\overparen{d_{u c}^{n}} / R\right)\right]\right\} \cdot\left(2 d_{n}^{S} d_{u c}^{S}\right)^{-1}\right)
$$

After that, the antenna radiation pattern needs to be selected to obtain antenna gain. With the same angular separation, antenna gain varies with the radiation pattern. Let $G_{j}(\theta)$ represent the antenna gain of the $j$ th spot beam, then

$$
G_{j}(\theta)=G_{M j} F_{j}^{2}(\theta)
$$

where $\theta$ is the angular separation from (1) or (5), $G_{M j}$ is the maximum gain of the $j$ th spot, and $F_{j}(\theta)$ is the normalized far-field radiation pattern. The radiation patterns have been modeled through suitable masks enveloping the maxima of the generic tapered-aperture antenna radiation pattern [7-8],

$$
F_{j}(\theta)=\hat{F}\left(u_{j}\right)=\frac{(p+1)(1-T)}{(p+1)(1-T)+T} \cdot\left[\frac{2 J_{1}\left(u_{j}\right)}{u_{j}}+2^{p+1} p ! \frac{T}{1-T} \frac{J_{p+1}\left(u_{j}\right)}{u_{j}^{p+1}}\right] \text { (7) }
$$

where: $u_{j}=\pi d_{a j} \sin \theta / \lambda ; d_{a j}$ is the effective aperture diameter of the jth spot; $\lambda$ is the wavelength; $J_{p}(u)$ is the

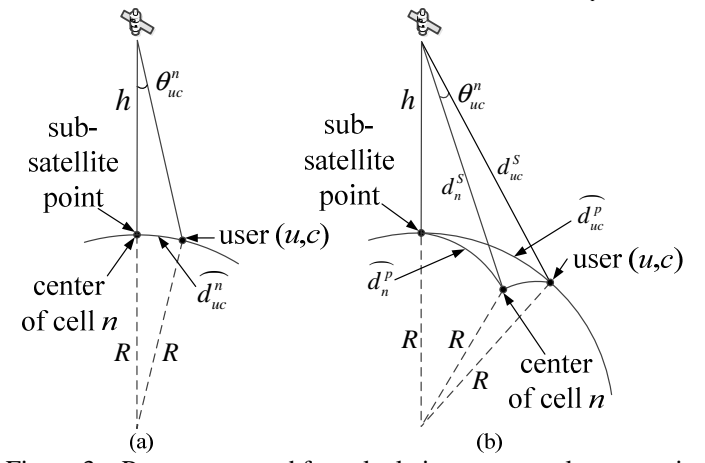

Figure 3. Parameters used for calculating user angular separation (a) Not considering IP (b) Considering IP 
Bessel function of the first kind and order $p ; T$ is the aperture edge taper.

The last part of interference modeling is to select a general $\mathrm{C} / \mathrm{I}$ formula to measure the interference level. The calculation of C/I is introduced in detail in Section IV.

\section{SimplificAtion OF C/I FORMUla FOR SATELLITE LINK}

For OFDMA system, it can be considered that no intrabeam interference exists and that all interference comes from other beams. Therefore, the interference considered in an OFDMA based GEO satellite system is IBI. Since OFDMA is a frequency and time division system, there's at most one interfering user existing in each other beam at a certain time and a certain frequency band. As for one or none interfering user existing in a spot beam, it depends on the layout pattern deployed, and whether the beam is full-loaded, as well as the resources scheduling strategy [10].

In multi-beam satellite systems, $\mathrm{C} / \mathrm{I}$ is often used to characterize interference. $\mathrm{C} / \mathrm{I}$ can be obtained by the calculation of the ratio of carrier power and IBI power. As shown in Fig. 4, $(m, n)$ represents the wanted user and the interfering user is represented by $(u, c)$.

\section{A. Up Link}

Using the OFDM up-link C/I formula introduced by Ref. 6 , the carrier power is

$$
C_{u p}=\left[w_{m n} g_{m n}\left(\alpha_{m n}\right) G_{n}\left(\theta_{m n}^{n}\right)\right] /\left[\left(4 \pi d_{m n} / \lambda\right)^{2} f_{m n}\left(\alpha_{m n}\right)\right]
$$

where: $w_{m n}$ is the power transmitted by user $(m, n) ; \alpha_{m n}$ is the angle between the tangent to the Earth in the location of user $(m, n)$ and the line between the satellite and user $(m, n)$, and $g_{m n}\left(\alpha_{m n}\right)$ is the terminal antenna gain of user $(m, n)$ in the direction of $\alpha_{m n} ; \theta_{m n}^{n}$ is the angle between the boresight of cell $n$ and the line between the satellite and user $(m, n)$, and $G_{n}\left(\theta_{m n}^{n}\right)$ is the satellite antenna gain in cell $n$ in the direction of $\theta_{m n}^{n} ; d_{m n}$ is the slant range for the link from user $(m, n)$ to the satellite; $\lambda$ is the wavelength; $f_{m n}\left(\alpha_{m n}\right)$ is the mobile channel fading experienced by user $(m, n)$ on the angle $\alpha_{m n}$.

The IBI power is calculated as

$$
I_{u p}=\sum_{\substack{c=1 \\\{\neq n}}^{N C} \frac{w_{u c} g_{u c}\left(\alpha_{u c}\right) G_{n}\left(\theta_{u c}^{n}\right)}{\left(4 \pi d_{u c} / \lambda\right)^{2} f_{u c}\left(\alpha_{u c}\right)} \cdot \mu_{u c} \rho_{c}^{n}
$$

where: $N C$ is the number of cells reuse the same frequency as cell $n ; \mu_{u c}$ is the activity factor of user $(u, c)$, which

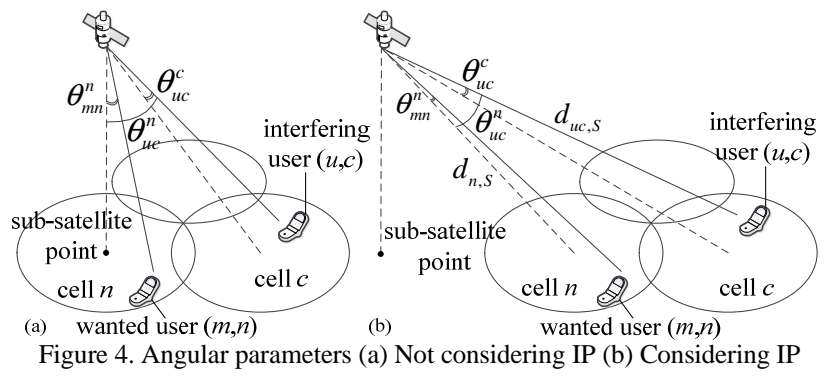

depends on the service utilized by the user; $\rho_{c}^{n}$ is the polarization isolation between cell $c$ and cell $n$.

Ref. 6 introduces the most general up-link C/I formula, but it is not simplified for a specific scenario. Assuming the power control for up link is ideal, each beam's power at the satellite receiver is the same, which is registered as $P_{\text {rec }}$. So for the wanted beam and the interfering beam,

$$
\begin{gathered}
{\left[w_{m n} g_{m n}\left(\alpha_{m n}\right) G_{n}\left(\theta_{m n}^{n}\right)\right] /\left[\left(4 \pi d_{m n} / \lambda\right)^{2} f_{m n}\left(\alpha_{m n}\right)\right]=P_{r e c}} \\
{\left[w_{u c} g_{u c}\left(\alpha_{u c}\right) G_{c}\left(\theta_{u c}^{u}\right)\right] /\left[\left(4 \pi d_{u c} / \lambda\right)^{2} f_{u c}\left(\alpha_{u c}\right)\right]=P_{\text {rec }}}
\end{gathered}
$$

Substitute (10) and (11) into (8) and (9), then

$$
\begin{aligned}
& C_{\text {up }}=P_{\text {rec }} \\
& I_{u p}=\sum_{\substack{c=1 \\
c \neq \neq}}^{N C} \frac{P_{r e c} G_{n}\left(\theta_{u c}^{n}\right)}{G_{c}\left(\theta_{u c}^{u}\right)} \cdot \mu_{u c} \rho_{c}^{n}
\end{aligned}
$$

Therefore, up-link $\mathrm{C} / \mathrm{I}$ is simplified as

$$
\left(\frac{C}{I}\right)_{u p}=\frac{C_{u p}}{I_{u p}}=\left[\sum_{\substack{j=1 \\ c \neq n}}^{N C} \frac{G_{n}\left(\theta_{u c}^{n}\right)}{G_{c}\left(\theta_{u c}^{u}\right)} \cdot \mu_{u c} \rho_{c}^{n}\right]^{-1}
$$

\section{B. Down Link}

Similar as up link, using the OFDM down-link C/I formula introduced by Ref. 6, the carrier power is

$$
C_{\text {down }}=\left[W_{n} g_{m n}\left(\alpha_{m n}\right) G_{n}\left(\theta_{m n}^{n}\right)\right] /\left[\left(4 \pi d_{m n} / \lambda\right)^{2} f_{m n}\left(\alpha_{m n}\right)\right]
$$

where $W_{n}$ is the power transmitted to cell $n$ by the satellite, and the definition of other parameters is same as above.

The IBI power is

$$
I_{\text {down }}=\sum_{\substack{c=1 \\ c \neq n}}^{N C} \frac{\mu_{u c} W_{c} g_{m n}\left(\alpha_{m n}\right) G_{c}\left(\theta_{m n}^{c}\right) \rho_{n}^{c}}{\left(4 \pi d_{m n} / \lambda\right)^{2} f_{m n}\left(\alpha_{m n}\right)}
$$

The most general down-link $\mathrm{C} / \mathrm{I}$ formula is introduced by Ref. 6, but it's not simplified for a specific scenario. Unlike up link, down link has no power control. Usually, the satellite transmits equal power to each cell, $W_{n}=W_{c}$. In down link, the desired signal and the interfering signals reach the same wanted user through the same path, so the transmission attenuation in free space $(4 \pi d / \lambda)^{2}$ and the channel attenuation $f(\alpha)$ are the same experienced by all the interfering signals and the desired signal. In addition, the antenna gain of mobile terminal $g(\alpha)$ is also the same. If the specific numerical values of the carrier power and the interference power are not required, these parameters don't need to be calculated in order to obtain C/I. As a result, down-link $\mathrm{C} / \mathrm{I}$ is simplified as

$$
\left(\frac{C}{I}\right)_{\text {down }}=G_{n}\left(\theta_{m n}^{n}\right) /\left[\sum_{\substack{c=1 \\ l \neq n}}^{N C} G_{c}\left(\theta_{m n}^{c}\right) \cdot \mu_{u c} \rho_{n}^{c}\right]
$$

\section{SimUlation AND ANALYSIS}

Taking the beam planning scheme shown in Fig. 2 for example, a comparison of C/I of users at the center of three chosen cells with and without the consideration of IP is made, based on the simulation using different FR factor. The simulation conditions are: the system is full-loaded; the power control for up link is ideal; $G_{M j}$ and $d_{a j}$ for each 
beam are the same; $\mu=1$ for each user; $\rho=1$ for the cells reuse the same frequency; $W$ is the same for each cell; $T=11 \mathrm{~dB}, p=2$, and $d_{a j}=13.17 \mathrm{~m}$. Other parameters used in the simulation are listed in Table I.

TABLE I. SIMULATION PARAMETERS

\begin{tabular}{|l|r|}
\hline \multicolumn{1}{|c|}{ Parameter } & \multicolumn{1}{|c|}{ Value } \\
\hline GEO satellite longitude & $110^{\circ} \mathrm{E}$ \\
\hline Number of spot beams & 88 \\
\hline Cell radius & $240 \mathrm{~km}$ \\
\hline Frequency & $2 \mathrm{GHz}$ \\
\hline Latitude and longitude of cell 1 & $25.3^{\circ} \mathrm{N}, 109.4^{\circ} \mathrm{E}$ \\
\hline Latitude and longitude of cell 2 & $35.0^{\circ} \mathrm{N}, 98.7^{\circ} \mathrm{E}$ \\
\hline Latitude and longitude of cell 3 & $41.7^{\circ} \mathrm{N}, 86.0^{\circ} \mathrm{E}$ \\
\hline
\end{tabular}

When adopting different FR schemes, central user C/I of each cell is

TABLE II. CENTRAL USER C/I (DB) OF CELL 1, 2 AND 3

\begin{tabular}{|c|c|c|c|c|c|}
\hline \multirow{4}{*}{$\begin{array}{c}\text { FR } \\
\text { factor }\end{array}$} & \multicolumn{2}{|c|}{ Not considering IP } & \multicolumn{2}{c|}{ Considering IP } \\
\cline { 2 - 6 } & 1 & Up-link & Down-link & Up-link & Down-link \\
\hline \multirow{4}{*}{ Cell 1 } & 0.07 & 1.98 & -1.17 & 0.14 \\
\cline { 2 - 6 } & 3 & 15.84 & 18.04 & 13.49 & 16.19 \\
\cline { 2 - 6 } & 4 & 20.02 & 21.27 & 18.55 & 19.85 \\
\cline { 2 - 6 } & 7 & 23.13 & 24.16 & 22.03 & 22.83 \\
\hline \multirow{4}{*}{ Cell 2 } & 1 & -0.45 & 1.23 & -2.94 & -1.98 \\
\cline { 2 - 6 } & 3 & 14.79 & 17.42 & 9.37 & 12.44 \\
\cline { 2 - 6 } & 4 & 17.85 & 19.25 & 13.92 & 16.00 \\
\hline \multirow{4}{*}{ Cell 3 } & 7 & 22.19 & 23.26 & 20.86 & 21.76 \\
\cline { 2 - 6 } & 1 & -0.59 & 0.98 & -4.26 & -3.52 \\
\cline { 2 - 6 } & 3 & 15.57 & 18.36 & 7.35 & 9.05 \\
\cline { 2 - 6 } & 4 & 18.12 & 19.93 & 11.84 & 14.22 \\
\hline
\end{tabular}

It can be seen from Table II that when not considering IP, C/I of each cell is slightly different. That is because when each cell is assumed to be the OP of the satellite, interference of each cell depends not on its own latitude and longitude, but on the number of interfering cells and the distance from those cells. When the FR factor increases, the number of interfering cells decreases and the distance gets larger, introducing less interference to the interfered cell.

However, when considering IP, all C/I has a certain degree of deterioration, and the larger the distance between the cell and the sub-satellite point is, the worse the $\mathrm{C} / \mathrm{I}$ becomes. That's because when the cell is near the subsatellite point, the shape of beam IP is slightly different from that of the OP, and the overlap between beams is small, introducing weak interference. While the cell is far from the sub-satellite point, the shape of beam IP is much different from that of the OP, and the overlap between beams is large, introducing the interfered cell strong interference.

Therefore, when planning satellite beams, if the position of each cell boresight is planned according to circular cell distribution, severe interference will be introduced. It is suggested to take IP into consideration when planning beams. An alternative is that the position of each cell boresight is planned according to elliptic cell distribution to make all beams cover the whole area and the overlap between beams as small as possible, which can reduce both the IBI and the number of beams.

\section{CONCLUSION}

This paper presents a complete process of interference modeling and analysis for inclined projective multiple beams of OFDMA based GEO satellite communication systems. Taking 3 typical cells for example, a comparison of $\mathrm{C} / \mathrm{I}$ of the central user with and without the consideration of IP is made, based on the simulation using different FR factor, and the corresponding analysis is presented. It can be seen that when taking IP into consideration, C/I of each cell has a certain degree of deterioration, and the larger the distance between the cell and the sub-satellite point is, the worse the $\mathrm{C} / \mathrm{I}$ becomes. This research can provide reference for later satellite beam planning.

\section{ACKNOWLEDGMENT}

This work is supported by the National Science Foundation of China (Grant No. NFSC \#61071083) and the National High-Tech Research and Development Program of China (863 Program), No. 2012AA01A506.

Corresponding author: Jianjun $\mathrm{Wu}$; phone: +86-1062752848; e-mail: just@pku.edu.cn.

\section{REFERENCES}

[1] H. W. Kim, T. C. Hong, K. Kang, B. J. Ku, S. Kim, and S. Yeo, “A Satellite Radio Interface for IMT-Advanced System Using OFDM,” International Conference on Information and Communication Technology Convergence (ICTC), 2010, pp. 303-308.

[2] S. Cioni, A. Vanelli-Coralli, G. E. Corazza, P. Burzigotti, and P.-D. Arapoglou, "Analysis and Performance of MIMO-OFDM in Mobile Satellite Broadcasting Systems,” IEEE Global Telecommunications Conference, 2010, pp. 1-6.

[3] H. Wang, W. Pan, and C. Pan, "The Research of OFDM Channel Estimation in Broadband Satellite Communication Systems," International Conference on Environmental Science and Information Application Technology, vol. 1, 2009, pp. 515-518.

[4] W. C. Y. Lee, “Overview of cellular CDMA,” IEEE Transactions on Vehicular Technology, vol. 40, No. 2, 1991, pp. 291-302.

[5] K. S. Gilhousen, I. M. Jacobs, R. Padovani, and L. A. Jr. Weaver, "Increased capacity using CDMA for mobile satellite communication," IEEE Journal on Selected Areas in Communications, vol. 8, No. 4, 1990, pp. 503-514.

[6] P. Loreti, and M. Luglio, "Interference evaluations and simulations for multisatellite multibeam systems," International Journal of Satellite Communications, vol. 20, No. 4, 2002, pp. 261-281.

[7] F. Vatalaro, G. E. Corazza, C. Caini, and C. Ferrarelli, "Analysis of LEO, MEO, and GEO global mobile satellite systems in the presence of interference and fading," IEEE Journal on Selected Areas in Communications, vol. 13, No. 2, 1995, pp. 291-300.

[8] C. Caini, G. E. Corrazza, G. Falciasecca, M. Ruggieri, and F. Vatalaro, "A spectrum- and power-efficient EHF mobile satellite system to be integrated with terrestrial cellular systems," IEEE Journal on Selected Areas in Communications, vol. 10, No. 8, 1992, pp. $1315-1325$.

[9] E. Lutz, "Issues in satellite personal communication systems", ACM Wireless Network, vol. 4, 1998, pp. 109-124.

[10] F. Meng, J. Chen, S. Ren, J. Guo, and J. Wu, "Comparison of Frequency Reuse Schemes in OFDMA based Multi-beam Satellite Communications", AIAA International Communications Satellite Systems Conference, 2011. 